\title{
CALIDAD EN LAS URGENCIAS HOSPITALARIAS. OPINIÓN Y SATISFACCIÓN DE LOS PACIENTES
}

José María Bleda García*

Uno de los objetivos generales que pretende alcanzar el Complejo Hospitalario y Universitario de Albacete es mejorar la calidad asistencial que se presta en el mismo. Para poder lograrlo, entre otras actuaciones, se considera que conocer la opinión y el grado de satisfacción de los usuarios es necesario.

Este estudio pretendía valorar la opinión y el grado de satisfacción de los pacientes atendidos en el Servicio de Urgencias del Hospital General de Albacete, con la finalidad de mejorar la calidad y obtener una visión más global del tipo de atención que se está efectuando desde el mismo.

El objetivo principal de la investigación era conocer qué pensaban los usuarios del Servicio de Urgencias sobre diferentes aspectos tales como: admisión, tiempo de espera, asistencia sanitaria, trato humano recibido, el entorno ambiental, y medir, a la vez, el grado de satisfacción respecto a ellos. Para ello hemos procurado ver las actitudes de los enfermos, las circunstancias de su llegada al servicio, el grupo que le introduce, que le atiende y se ocupa de él (de sus papeles, de su asistencia, de su intimidad), su patología, los testimonios de los enfermos o de los familiares (reclamaciones, sugerencias), así como su perfil socioeconómico y su nivel de satisfacción o insatisfacción.

* Doctor en Sociología. Profesor de Sociología de la Universidad de Castilla-La Mancha. Miembro de la Asociación Castellano-Manchega de Sociología. 


\section{METODOLOGÍA}

El instrumento que hemos utilizado para medir la opinión y el grado de satisfacción de los usuarios ha sido una encuesta, que se elaboró teniendo en cuenta las aportaciones de otros estudios de estas características. Este cuestionario se envió por correo a una muestra de pacientes seleccionada (2000) entre todos los usuarios que habían sido atendidos en el mes de noviembre del año 2000 (8089) en el Servicio de Urgencias del Hospital General de Albacete y a la que contestaron 490 individuos, es decir un $24,5 \%$, porcentaje similar a estudios de este tipo. A los encuestados se les facilitó la devolución de la encuesta adjuntándoles un sobre que, a la vez, garantizaba su anonimato. No se recordó la contestación a los que no lo hicieron y se incidió en la voluntariedad de las respuestas. En la elaboración del cuestionario han participado profesionales de los servicios de: Informática, Dirección Médica, Docencia e Investigación y Gerencia.

El proceso de recogida de información se realizó a través del cuestionario que contenía 34 preguntas con varias opciones de respuesta, de ellas solamente dos eran preguntas abiertas. Además se recogieron al principio del cuestionario unos datos socioeconómicos de interés para el estudio como: sexo, edad, residencia habitual, situación laboral y nivel de estudios.

El cuestionario se dividía en siete apartados: indicadores sociales (ítems 15), admisión (i. 6-11), tiempo de espera (i. 12-16), asistencia sanitaria (i. 17-23), trato humano (i. 24-28), medio ambiente (i. 29-34) y satisfacción general (i. 35-39).

\section{RESULTADOS}

\section{Indicadores sociales}

El cuestionario lo ha respondido una mayoría de mujeres $(51.8 \%)$ y un $46.5 \%$ de hombres.

La edad de los encuestados se concentra, sobre todo, en los comprendidos entre 26 a 65 años, que suponen el $49.5 \%$ y los mayores de 65 años, que representan el $27.1 \%$. La edad media es de 45 años.

El 51.6\% reside en Albacete capital, el 35.1\% en la provincia de Albacete, el $9 \%$ de otras provincias de Castilla-La Mancha y un 1.6\% de fuera de la comunidad autónoma.

La gran mayoría de los encuestados no trabaja (64.1\%), sólo lo hace un 32\%.

El nivel de estudios es el siguiente: un $24.1 \%$ no tiene estudios, un $41.4 \%$ tiene estudios primarios, un $18.6 \%$ estudios secundarios y un $10.4 \%$ estudios universitarios.

\section{Admisión}

En primer lugar, destacar que un elevado número de los encuestados (74.9\%) 
responde que ha elegido este servicio de urgencias porque no tenía posibilidad de utilizar otro.

Los motivos que expresan para ir al servicio de urgencias son por orden prioritario los siguientes: se lo indicó su médico de cabecera (39.8\%), confianza en el servicio (27.3\%), proximidad (11.2\%), le enviaron de otra institución (12.4\%), no le dan cita en el especialista (4.9\%) y no le atendieron en el centro de salud (3.5\%).

Otro dato a destacar en el primer contacto que tienen al llegar al servicio de urgencias es, que un $37.1 \%$ de los usuarios manifiesta que no había ningún celador que le orientase a dónde dirigirse ni que hacer, ahora bien cuando este profesional estaba están muy satisfechos con la atención que recibieron: normal un $34.7 \%$, satisfecho un $33.4 \%$ y muy satisfecho un $19.3 \%$. No obstante, hay un $12.6 \%$ de insatisfechos.

El problema por el que acudieron al servicio de urgencias lo consideran muy importante, pues más de un $90.8 \%$ de los usuarios opinan que el problema era importante y muy importante.

Los trámites burocráticos con los que se encuentran en admisión estiman que son normales y sencillos de realizar.

\section{Tiempo de espera}

El tiempo medio que tuvieron que esperar desde que llegaron al servicio de urgencias hasta que le atendieron para su clasificación fue de 31 minutos, si bien habría que especificar que un $14.1 \%$ fue atendido antes de 5 minutos, un $32.0 \%$ entre 6 y 15 minutos, un $20.2 \%$ entre 16 y 30 minutos, y un $18.6 \%$ más de media hora.

El tiempo medio de espera desde que llegaron hasta que le atendieron de su dolencia fue de 70 minutos, aunque un $41.1 \%$ fue atendido antes de media hora, el $19.4 \%$ entre media y una hora, y un $25.3 \%$ tuvo que esperar más de una hora.

Ese tiempo de espera es considerado por un $40.8 \%$ como demasiado, un $40.2 \%$ como normal y un 17.6 como corto.

El tiempo medio transcurrido desde que llegó hasta que se marchó a su domicilio o ingresó en el Hospital fue alrededor de tres horas. Destaca el 16.3\% de los que estuvieron más de cuatro horas. Sólo un $20.4 \%$ estuvieron menos de una hora.

Consideran muy importante que alguien se interese por su situación cuando llegan al servicio de urgencias y que le orienten sobre el tiempo que tienen que esperar para ser atendidos. Así opinan el 89.2\%: un 58.6\% dicen que es muy importante y un $30.6 \%$ que es importante.

\section{Asistencia sanitaria}

El $89.4 \%$ de la población consultada se muestra satisfecha con la atención sanitaria recibida: normal un $17.8 \%$, satisfecha un $42.4 \%$ y muy satisfecha un $29.2 \%$. 
La valoración de la capacidad profesional de los médicos y del personal de enfermería es muy alta, pues para los primeros los resultados obtenidos son: $16.9 \%$ normal, $39.6 \%$ buena y $38.4 \%$ muy buena, y para los segundos: $21.0 \%$ normal, $40.4 \%$ buena y $32.2 \%$ muy buena. Sólo un 3.9\% (médicos) y un 5.3\% (enfermería) consideran que la capacidad de estos profesionales es mala y muy mala.

A la mayoría de los pacientes que le realizaron alguna prueba diagnóstica la explicación que les dieron fue clara en un $71.2 \%$, no así para un $14.1 \%$.

En esa misma línea de comprensión se manifiestan el $88.6 \%$ de los pacientes cuando respondieron a la pregunta de sí comprendieron la información que le dieron en el informe de alta sobre el tratamiento a seguir en casa. Si bien, un $4.9 \%$ manifiesta que no entendió la explicación.

En general, los usuarios están muy satisfechos con las explicaciones que le dio el personal médico sobre su enfermedad: normal un 19.4\%, satisfecho un $46.1 \%$ y muy satisfecho un $23.9 \%$. No obstante, un $8.8 \%$ se encuentra poco satisfecho $(8.0 \%)$ y muy insatisfecho $(0.8 \%)$.

\section{Trato humano}

El interés que mostró el personal sanitario para entender y comprender el problema fue muy positivo pues el $27.1 \%$ de los pacientes respondió que el interés fue normal, un $41.2 \%$ que fue bueno y un $26.9 \%$ muy bueno. Un $3.9 \%$ opinó que el interés fue malo y muy malo.

También valoran muy positivamente la disposición del personal sanitario para ayudarle cuando lo necesitó: un $27.6 \%$ lo valoró normal, un $46.3 \%$ buena y un $21.2 \%$ muy buena. Un $4.3 \%$ estimó una disposición negativa, considerándola un $3.5 \%$ como mala y un $0.8 \%$ muy mala.

En cuanto a la seguridad que transmite el personal sanitario a los pacientes, estos opinan: que es normal un $25.1 \%$, buena un $48.6 \%$ y muy buena un $17.3 \%$. Un $7.9 \%$ estiman que la seguridad que les transmiten es mala (6.3\%) y muy mala (1.6\%).

La amabilidad y el respeto con el que les trataron son valorados muy positivamente, ya que el $24.9 \%$ lo valora normal, el $43.1 \%$ buena y el $26.9 \%$ muy buena. Sin embargo, un $4.7 \%$ estima lo contrario.

En general, el trato humano recibido por los pacientes es valorado positivamente por el $95.3 \%$ de ellos: $25.9 \%$ normal, $43.5 \%$ bueno y $25.9 \%$ muy bueno. Un $4.1 \%$ opina que el trato humano recibido fue negativo.

\section{Medio ambiente}

Las indicaciones que encontraron para orientarse al llegar y saber a dónde ir las consideran en un alto porcentaje (92.7\%) adecuadas: normales el $27.8 \%$, buenas el $48.0 \%$ y muy buenas el $16.9 \%$. Un $5.9 \%$ las consideró malas $(5.3 \%)$ y muy malas $(0.6 \%)$. 
Respecto a la satisfacción del grado de confort en la sala de espera destaca que un $29.4 \%$ de los encuestados opina que está poco satisfecho (21.4\%) y muy insatisfecho $(8.0 \%)$, si bien un $68.6 \%$ consideró confortable la sala de espera.

La valoración negativa respecto al medio ambiente también se destacó cuando opinaron sobre el ambiente de la sala de espera (ruido, tranquilidad, aglomeración..), pues un $31.2 \%$ expresó su malestar. Un $46.1 \%$ lo consideró normal, un $14.1 \%$ bueno y un $6.3 \%$ muy bueno.

Sobre la garantía de su intimidad el $78.2 \%$ se expresó positivamente, mientras que un $18.8 \%$ lo hizo contrariamente.

El estado en que se encontraban las instalaciones de urgencia (salas de espera, consultas, aseos,..) era normal para el $41.8 \%$, bueno para el $34.1 \%$ y muy bueno para el $8.4 \%$. Un $14.4 \%$ estima que las condiciones eran malas y muy malas.

Mejorarían las instalaciones de urgencia en los aspectos de comodidad (47.8\%), de intimidad (29.4\%) y de temperatura (8.6\%).

\section{Satisfacción general}

Un $90.8 \%$ se declara satisfecho en cuanto a la resolución de su problema por el que acudió a Urgencias, un 7.3\% no lo considera así.

Esta mayoritaria opinión también es refrendada cuando responden a la cuestión de que expresen su satisfacción de los cuidados sanitarios recibidos, pues un $91.2 \%$ así lo manifestaron: $26.5 \%$ normal, $45.7 \%$ satisfecho y $19.0 \%$ muy satisfecho. La opinión negativa está representada por un $7.7 \%$.

Satisfacción que es apoyada cuando ante la pregunta de sí recomendaría este servicio de urgencias a otras personas un $74.1 \%$ responde que no lo dudaría, mientras que un $19.8 \%$ tendría dudas y un $2.9 \%$ no lo haría nunca.

En general, la mayoría de los pacientes atendidos en el servicio de urgencias $(88.8 \%)$ considera aceptable su estancia en él: $23.1 \%$ normal, $44.1 \%$ aceptable y $21.6 \%$ muy aceptable. Por contra, un $10.6 \%$ no lo estima aceptable: $8.4 \%$ poco aceptable y $2.2 \%$ muy inaceptable.

Al finalizar el cuestionario se les preguntaba a los usuarios sobre qué mejoras se debían hacer en el Servicio de Urgencias, y las sugerencias que realizaron se centraban mayoritariamente en tres aspectos: reducir el tiempo de espera, mejorar la confortabilidad de la sala de espera (mayor comodidad, mejorar la limpieza, adecuar temperatura ambiente) y aumentar el personal sanitario. Aunque también incidían en otros dos: mejorar el trato humano (sobre todo la amabilidad) y aumentar la información al paciente mientras espera.

\section{CONCLUSIONES}

La mayoría de las respuestas han sido de mujeres, pero muy similares a las de los hombres. También la mayoría es población en edad laboral, residente en la capital, amas de casa o que no trabajan y con estudios primarios. 
Una gran mayoría declara que no tenía otra posibilidad de ir a otro servicio de urgencias, lo que puede suponer o bien un desconocimiento de los otros servicios de urgencia que hay en la ciudad y en la provincia, o bien que tienen una mayor confianza en las urgencias del hospital. En cuanto a los motivos que expresan del porqué han ido al Servicio de Urgencias del Hospital, resalta que un $4.9 \%$ es porque no le dan cita en el especialista y un 3.5\% porque no le atendieron. En el primer contacto que se tiene al llegar al Hospital, se dan bastantes momentos en que no hay un celador que les oriente sobre lo que tienen que hacer. Cuando lo hay los usuarios se encuentran satisfechos con la atención recibida. Sea o no urgente el motivo asistencial por el que acuden, casi todos consideran que su problema es importante. Las tareas burocráticas ante las que se encuentran en admisión no tienen dificultad en realizarlas.

El tiempo de espera es uno de los aspectos que más insatisfacción produce, pues un $40.8 \%$ considera que es demasiado el que han tenido que esperar, un $16.3 \%$ estuvo en urgencias más de cuatro horas. El $89.2 \%$ cree conveniente que haya una persona que les oriente sobre el tiempo que tienen que esperar para ser atendidos.

Se da una gran satisfacción $(89.4 \%)$ ante la atención sanitaria recibida.Valoran en un alto grado la capacidad profesional tanto del personal médico como del personal de enfermería. La comprensión a la hora de las explicaciones sobre pruebas diagnósticas y sobre el tratamiento a seguir es muy alta, no obstante a un $14.1 \%$ no le explicaron los resultados de las pruebas que le hicieron y un 4.9\% no entendió la información que se les dio. En general, están satisfechos con las explicaciones médicas sobre su enfermedad, si bien un $8.8 \%$ se encuentra insatisfecho.

Existe una valoración muy positiva (95.3\%) ante el trato humano que les ha dispensado el personal del servicio de urgencias en diferentes aspectos, tales como: el interés que mostró el personal sanitario ante la enfermedad del usuario, su disposición a ayudarle en cuanto lo necesita, la seguridad que les transmitió, y la amabilidad y el respeto con que lo trataron. No obstante, alrededor de un $4 \%$ de los usuarios se encuentra muy insatisfecho en el trato que han recibido de los profesionales sanitarios.

En los aspectos medioambientales es donde han expresado un alto grado de insatisfacción, pues una buena parte de los usuarios (sobre el 25\%) estiman que el grado de confort de la sala de espera, el ambiente existente en ella y el estado de las instalaciones no es el deseable. Una parte importante de los usuarios (18.8\%) consideran que no se ha garantizado su intimidad. Para ello proponen mejorar las instalaciones sobre todo en los aspectos de comodidad y de intimidad.

Existe una opinión mayoritaria (90\%) con relación a la satisfacción sobre la resolución de su problema, sobre los cuidados sanitarios recibidos y sobre su estancia en general en el Servicio de Urgencias. Si bien, una vez más, se contrasta que entre un $7 \%$ y un $10 \%$ de los usuarios atendidos se encuentran insatisfechos. 


\section{DISCUSIÓN}

Habría que establecer cauces que derivaran a los centros de salud las urgencias que pueden ser tratadas en ellos, para lo que es necesario una mayor coordinación con el área de atención primaria.

Se tendría que estudiar detenidamente el caso de los pacientes que acuden a urgencias porque no se les da cita en el especialista.

Debería incidirse más en la presencia continua de celadores en la puerta de urgencias y en la posibilidad de la existencia de algún trabajador que acogiese a los pacientes y les orientase sobre el tiempo que van a tener que esperar para ser atendidos.

Es necesario reducir el tiempo de espera, tanto en la clasificación como en la atención sanitaria. Actuación que probablemente supondría un aumento de la plantilla del personal sanitario, una mayor implicación de los especialistas que se encuentren de guardia o una nueva reorganización del servicio.

Es urgente remodelar la sala de espera de urgencias para que exista una mayor comodidad y confortabilidad.

Hay que preservar aún más la intimidad de los pacientes cuando sean atendidos por el personal sanitario. 


\section{BIBLIOGRAFÍA}

ARANAZ, J., MIRA, J.J., y RODRIGUEZ, J., «La satisfacción de los profesionales como un aspecto más del control de calidad de los hospitales, Todo Hospital, 1988; 47: 53-60.

BELZUNEGUI, M.A., et al., «Tiempos de espera en un servicio de urgencias como indicador de calidad asistencia», Emergencias , 1993; 3: 84-89.

BENAVIDES, F.G., et al., «Aplicación y validez de un cuestionario para medir la satisfacción de los usuarios de un centro de salud», Atención Primaria, 1987; 2: 91-95. BLEDA GARCÍA, J.M., «Estudio de opinión y satisfacción de los pacientes atendidos en el servicio de urgencias del Hospital General de Albacete (España)», Gestión Hospitalaria, 2000; 11(2): 51-57.

COMÍN, E., «Validación de encuestas», Atención Primaria 1990; 5: 386-390.

DEL CASTILLO, M., et al. «Estudio del área de urgencias de un hospital general. Grado de adecuación de las visitas», Medicina Clínica 1986; 13: 23-26.

FREIRE, J.M., «Las preferencias del consumidor en los servicios sanitarios. Nivel práctico», Mapfre Medicina, 1991; 3: 141-150.

GARCÍA, S., y CUSTEY, M., «Evaluación de la satisfacción de los profesionales de atención primaria», Medifam 1992; 1: 16-24.

HELGUERA, F., «Grado de satisfacción de los profesionales de los servicios de urgencia», Todo Hospital ,1995; 114: 63-73.

IBAÑEZ, R., «Estudio de la satisfacción de los pacientes atendidos en un servicio de urgencias», Calidad Asistencial 1995; 2: 60-63. «Valoración de la calidad asistencial en pacientes ingresados en la sala de observación de urgencias»,Emergencias 1992; 6: 249-253.

IBERN, P., «La mesura de la satisfacció en els serveis sanitaris», Gaceta Sanitaria 1992; 6: 176-185.

LLORET, J.et al., «Urgencias médicas. Análisis de 18.240 pacientes atendidos en el servicio de urgencias de un hospital general en el periodo de un año», Medicina Clínica 1984; 4: 135-141.

MARTÍNEZ, J., et al., «Razones del usuario en la utilización de un servicio de urgencias hospitalario», Clínica Española, 1992; 191: 488-493.

MIRA, J.J. et al., «¿Qué hace que los pacientes estén satisfechos?. Análisis de la opinión de pacientes y profesionales mediante la técnica Delphi», Calidad Asistencial, 1999; 14: 165-177.

MOZOTA, J.R., «Resumen de la encuesta de satisfacción del personal en el Hospital de Valdecilla», Todo Hospital,1990; 67: 21-28.

OTERO, P., y otros, «Estudio del Servicio de Urgencias del Hospital General "Río Carrión" de Palencia», Emergencias, 1991; 3(1): 58-68.

RUBIO, R.,»Función del control de calidad en la estructuración del servicio de urgencias del hospital», Todo Hospital,1992; 90: 33-39. «Calidad asistencial. Opinión de los usuarios», Todo Hospital, 1988; 47: 61-65. 
SERRANO, S., et al., «Trabajo social en urgencias», Emergencias, 1996; 4: 387-400.

SOLER, W., et al., «Tiempos de espera en urgencias. Control de calidad interno y externo», Todo Hospital, 1996; 129: 7-13. 